\title{
"Políticas públicas para la institucionalización de las redes de conocimiento en las Instituciones de Educación Superior en México, desde fines de los noventa ${ }^{1 /}$
}

\author{
María Teresa de Sierra Neves ${ }^{2}$ \\ Universidad Pedagógica Nacional (México) \\ Grupo de investigación HISULA \\ teredesierra@gmail.com
}

Recepción: 17/04/2013

Evaluación: 22/10/2013

Aceptación: 13/12/2013

Artículo de Reflexión

DOI: http:/ / dx.doi.org/ 10.9757/Rhela.22/11

RESUMEN

En este trabajo se analizan las políticas públicas en ciencia y tecnología que se aplicaron en México desde fines del siglo XX para conocer el impacto que ellas tuvieron en la promoción y desarrollo de las redes académicas como nuevos modos de organización, gestión y transferencia del conocimiento. Particularmente, interesa la interacción entre los cambios provocados por dichas políticas, en la configuración redes de grupos y centros de investigación, y el desarrollo de redes académicas como un fruto de la evolución natural de la actividad científica disciplinaria e interdisciplinaria.

En el trabajo se distinguen las siguientes dimensiones relevantes: la internacionalización del conocimiento resultado de la complejización de los saberes; el contexto de nuevas regulaciones en que se desarrollan las formas de organización del conocimiento en las universidades; el contex- to histórico con nuevas demandas de la sociedad y los organismos públicos.

El enfoque metodológico del estudio es el del nuevo institucionalismo, y la estrategia de investigación se ha basado en las revisiones documentales y entrevistas a actores calificados. La principal conclusión del trabajo es que el efecto en la generación de redes de conocimiento por la aplicación de estas políticas no es homogéneo. Está mediado por las condiciones institucionales e históricas de cada institución y culturas disciplinarias.

Palabras clave: Revista Historia de la Educación Latinoamericana, redes de conocimiento, IES, CONACYT, PROMEP, internacionalización de las universidades, Nuevas regulaciones, institucionalización, practicas disciplinarias, imaginarios.

1 Este texto es una primera versión de uno de los capítulos del Proyecto de Investigación "Políticas públicas para la configuración de redes epistémicas en México. Estudio de caso de la UNAM". El proyecto lo estoy desarrollando en el Posdoctorado en Ciencias de la Educación, en la Universidad Pedagógica y Tecnológica de Colombia 2013-2014, El texto se articula al proyecto de investigación Reformas y movimientos universitarios en Colombia SGI 1295, desarrollado por el grupo Ilustración en América Colonial - ILAC y financiado por la Dirección de Investigaciones de la Universidad Pedagógica y Tecnológica de Colombia.

2 Doctora en Ciencias Políticas y Sociales. Orientación sociología. Facultad de Ciencias Políticas y Sociales de la UNAM, Estudiante de Posdoctorado de la Universidad Pedagógica y Tecnológica de Colombia, Maestría en Historia y Etnohistoria en la Escuela Nacional de Antropología e Historia (ENAH), Maestría en la Facultad Latinoamericana de Ciencias Sociales (FLACSO) Licenciatura en Sociología, Facultad de Ciencias Sociales de la Universidad de la República de Uruguay, adscrita actualmente a la Universidad Pedagógica Nacional, integrante del grupo de investigación HISULA de la Universidad Pedagógica y Tecnológica de Colombia. 
Public policies for the institutionalization of knowledge networks in the IES in Mexico, since the end of $90 \mathrm{~s}$

\section{ABSTRACT}

In this paper we analyze public policies in science and technology that were applied in Mexico since the late twentieth century; the impact that they had on the promotion and development of the academic networks as new way of organization, management and knowledge sharing.

Particularly, it is interested in the interaction between the changes caused by these policies, in the configuration of group networks and research centers, and the development of academic networks as a result of natural evolution of the scientific disciplinary and interdisciplinary activity.

In the work are distinguished the following relevant dimensions: the internationalization of knowledge, as a result of the complexity of the knowledge; the context of new regulations in which they develop ways of organization of knowledge in universities; the historical context with new demands of society and government agencies.

The methodological approach of the study is that of the new institutionalism, and the research strategy has been based on literature reviews and interviews with qualified operators.

The main conclusion of the work is that the effect on the generation of knowledge networks for the implementation of these policies is not homogeneous. It is mediated by the institutional and historical conditions of each institution and disciplinary cultures.

Key words: Journal History of the Latin American education, knowledge networks, IES, CONACYT, PROMEP, internationalization of universities, new regulations, institutionalization, disciplinary practices, imaginary.
Políticas públicas para a institucionalização das redes de conhecimento nas Instituições de Ensino Superior do México, desde fins dos 90

\section{RESUMO}

Neste trabalho se analisam as políticas públicas em ciência e tecnologia que se aplicaram no México desde fins do século XX para conhecer o impacto que elas tiveram na promoção e desenvolvimento das redes acadêmicas como novos modos de organização, gestão e transferência do conhecimento. Particularmente, interessa a interação entre as mudanças provocadas por interesses políticos, na configuração de redes de grupos e centros de pesquisa, e o desenvolvimento de redes acadêmicas como fruto da evolução natural da atividade científica disciplinar e interdisciplinar.

No trabalho se distinguem dimensões relevantes: a internacionalização do conhecimento resultado da complexidade dos saberes; o contexto de novas regulações em que se desenvolvem as formas de organização do conhecimento nas universidades; o contexto histórico com novas demandas da sociedade e os organismos públicos.

O enfoque metodológico do estudo é o do novo institucionalismo, e a estratégia de pesquisa foi baseada em revisões documentais e entrevistas a atores qualificados.

A principal conclusão do trabalho é que o efeito na geração de redes de conhecimento pela aplicação destas políticas não é homogêneo. Está mediado pelas condições institucionais e históricas de cada instituição e culturas disciplinares.

Palavras-chave: Revista História da Educação Latino-americana, redes de conhecimento, IES, CONACYT, PROMEP, internacionalização das universidades, Novas regulações, institucionalização, práticas disciplinares, imaginários. 


\section{INTRODUCCIÓN}

En este trabajo se busca analizar en qué medida la configuración de redes de conocimiento ${ }^{3}$ y su relación con el sector social y productivo son respuesta a las demandas actuales de las políticas públicas en ciencia y tecnología, a nivel nacional e internacional, para la generación, gestión y transferencia del conocimiento, o son resultado de una evolución natural de la actividad científica disciplinaria, que lleva a los investigadores a articularse en redes disciplinarias e interdisciplinarias, o son resultado de la conjunción de ambas.

Para tales efectos, se distinguen las distintas dimensiones que intervienen en este proceso, como lo son, en primer lugar, el papel importante que cumple la internacionalización del conocimiento, a consecuencia de la complejización -especialización de los saberes e interacción de los campos del conocimiento en una sociedad globalizada-. En segundo lugar, se considera el contexto histórico en que se desarrollan dichas formas de organización del conocimiento en las universidades, como es el tema de las nuevas regulaciones por parte de Consejo Nacional de Ciencia y Tecnología, (CONACYT), Programa de Mejoramiento del Profesorado (PROMEP) y la Secretaria de Educación Pública (SEP) y su relación con el sector social y productivo. En tercer lugar, se analiza cómo en el contexto histórico actual, en el que se han incrementado las demandas por parte de la sociedad y organismos públicos nacionales e internacionales, las prácticas investigativas que anteriormente se habían llevado la mayoría de las veces por una iniciativa o interés personal o de un grupo, en la actualidad se empiezan a incluir en los marcos institucionales, en paralelo con el tema de la internacionalización dentro de los planes estratégicos de las Instituciones de Educación Superior (IES).

Esta reflexión acerca de la construcción de redes de conocimiento busca analizar la configuración y o consolidación de grupos de investigación o redes de conocimiento, en torno a líneas de generación de conocimiento

3 Jaim Royero, “Gestión de sistemas de investigación en América Latina”, Revista Iberoamericana de Educación, (2007): 11-18. Las redes de conocimiento son interacciones humanas en la producción, almacenamiento, distribución, transferencia, acceso y análisis de los conocimientos producidos por la investigación o por el propio interés individual o colectivo por compartir información y conocimiento por medio de cualquier medio (electrónico o digital). Ver Jaim Royero, "Gestión de sistemas de investigación en América Latina”, Santiago de Chile Revista Iberoamericana de Educación, (2007): pág. 11-18.Consulta noviembre 2013 http://www.monografias.com/trabajos19/redes-conocimiento/redesconocimiento.shtml\#defin\#ixzz2LqAQg8KT 
a partir de políticas públicas como las impulsadas por CONACYT y PROMEP, tratando de evaluar en qué medida estas han contribuido a generar una cooperación efectiva en la actividad de investigación, así como a la producción de conocimiento científico. Este problema nos lleva a cuestiones de tipo epistemológico sobre cómo se construye el conocimiento en contextos institucionales y qué lugar ocupa dentro de ello las líneas y redes mencionadas.

Partiendo de que el desarrollo del conocimiento se cumple en un proceso social, en el marco de un sistema institucional, estas políticas asumen la propuesta de que cumplir ese proceso con base en definir líneas generales del conocimiento en el marco de redes epistémicas permite identificar y elaborar mejor los objetos de estudio, y con ello incrementar los resultados de la actividad científica por medio de que esta se cumpla, no de manera aislada sino mediante grupos de investigación. Esto supone una hipótesis epistemológica, en la que se postula que un objeto de estudio puede estudiarse más adecuadamente si se lo aborda desde distintos ángulos por parte de diferentes investigadores coaligados en torno a una línea de conocimiento que comparten en relación a dicho objeto, y nos remite a preguntarnos si el trabajo colectivo nos llevaría a un estudio de más calidad o con mejores resultados que el trabajo individual, y de qué manera podemos comprobar ese resultado.

Parecería que las actuales políticas de promoción de redes académicas implementadas por PROMEP y CONACYT son respuesta a experiencias previas, donde las estrategias de las políticas públicas se centraron en la evaluación y los incentivos económicos individuales. ${ }^{4}$

Todo esto motivado por el tipo de evaluación del trabajo personal y por el hecho de que esta estuvo vinculada al otorgamiento de estímulos económicos. Además de logros cuantitativos, dichas políticas generaron grandes tensiones en las comunidades académicas, ya que, por un lado, tuvieron de positivo que se promovió la profesionalización de las trayectorias académicas, pero paradójicamente provocaron una desinstitucionalización de la actividad de los mismos y por otro lado estimularon comportamientos individualistas.

4 María Teresa de Sierra Neves, Clarososcuros de la Profesionalización Académica. Estudio de caso la UNAM y la UAM (México: Editorial UPN y Doctorado en Educación de la Ciudad de México, 2007). 
Es por ello que, dándole continuidad a dicho tema, se considera relevante estudiar la interrelación de todos los factores: políticas públicas, actores, imaginarios, historia institucional, disciplina y generación en el análisis de la redes epistémicas.

Durante muchos años, la cooperación entre grupos de investigación, sobre todo en las Ciencias sociales, no constituía una prioridad en los planes disciplinarios ni institucionales para el desarrollo de las prácticas científicas en las instituciones de educación superior (IES). Según algunas investigaciones previas realizadas por mí, el perfil disciplinario había marcado las diferencias en la configuración de los grupos de investigación. En el caso de los físicos, biólogos, químicos fue siempre más común el trabajo colectivo, por la especificidad de la disciplina que requiere el trabajo en equipo. En cambio, en el caso de las ciencias sociales, si bien algunas veces se habían llevado a cabo, el trabajo en grupo fue casi siempre por la propia iniciativa e interés personal de los investigadores.

Por ello, resulta tan relevante, considerar las estrategias que se plantean como política pública e institucional en los noventa en México sobre las nuevas regulaciones para la configuración de redes de conocimiento, ya que PROMEP y CONACYT han tenido distintas modalidades de regulación de las actividades de investigación. Como resultado de esto es importante observar el papel de las historias institucionales y culturas disciplinarias a efecto de detectar el peso de las mediaciones culturales en las diferentes IES, así como en el desarrollo de la profesión académica y la conformación y consolidación de las redes de conocimiento. Muchos estudiosos del tema y algunos testimonios logrados por mí, ${ }^{5}$ en investigaciones realizadas con anterioridad, dan cuenta de cómo las cosmovisiones de los académicos e investigadores de distintos campos del conocimiento apuntan a considerar diferencias significativas respecto de la concepción de la profesión y / o carrera académica así como al proceso de desarrollo de redes de conocimiento.

Los debates sobre este tema versan en general acerca de si las diferencias de percepción sobre de cuáles deben ser las características sustantivas de la profesionalización académica y de la organización en grupos de investigación, es decir, si estas se corresponden con diferentes representaciones o imaginarios

5 María Teresa de Sierra y María Cristina Vera, "Proyecto de Investigación: Nuevas identidades profesionales y académicas. Discursos e imaginarios en el Horizonte histórico-contemporáneo”, (México: UNAM-UNC, 2008). 
disciplinarios acerca de las modalidades de producción del conocimiento. O si tales debates se refieren a diferentes grados de desarrollo evolutivo dentro de un mismo modelo de profesionalización académica. ${ }^{6}$

La hipótesis que se busca argumentar en este trabajo es la siguiente es si la evolución de las trayectorias de los grupos académicos hacia la profesionalización académica y la conformación de colectivos y redes de conocimiento en el marco de las nuevas política públicas para la educación superior y en ciencia y tecnología son resultado de la interacción de tres factores básicos.

De una parte, los grandes retos que se plantean para el desarrollo de la investigación científica, en la sociedad del conocimiento, como consecuencia de la complejización-especialización de los saberes e interacción de los campos del conocimiento en una sociedad globalizada; de la otra, las políticas de internacionalización de las universidades y finalmente las orientaciones de las políticas públicas que están dirigidas a promover redes epistémicas y su aplicación en las instituciones.

Esta exposición la hemos organizado en torno a tres grandes apartados. En el primero, plantearemos algunos antecedentes respecto del tema de exposición; en el segundo consideraremos nuestra perspectiva metodológica, y, en tercer lugar, revisaremos las implicaciones de las diferencias disciplinarias en la concepción de las redes. Finalizamos con algunas consideraciones generales y conclusiones.

Los interrogantes que orientan el presente trabajó son los siguientes: ¿Cuáles son las posibilidades reales que tienen los académicos de adaptar y reorientar sus trayectorias en función de la construcción de redes de conocimiento? ¿Cuáles son los límites a la plasticidad de estas trayectorias? ¿Cuál es el papel que les corresponden, pueden o están dispuestos a jugar los académicos en estos procesos?

De la revisión de la literatura, se observa que el tema de la construcción de redes epistémicas y el tema de los grupos académicos está cobrando cada vez mayor relevancia en el ámbito nacional, no obstante ello, desde la perspectiva del nuevo institucionalismo ha sido poco desarrollado, lo que justifica el

6 Silvio Leonardo Vacarezza. "Heterogeneidad en la conformación de la Profesión Académica: una Comparación entre Químicos y Sociólogos”, Redes 13, 26 (2007): 17. 
interés por el tema. Todo esto motivado por el tipo de evaluación del trabajo personal y por el hecho de que esta estuvo vinculada al otorgamiento de estímulos económicos.

En relación al tema específico sobre redes epistémicas, constituyen de gran interés los trabajos de Rocío Grediaga Kuri sobre "La carrera académica, disciplinas y organizaciones. Procesos de socialización académica y sus efectos en las actividades y resultado de los académicos mexicanos". Realiza un análisis de las tradiciones disciplinarias, y las redes como elementos clave del proceso de comunicación del conocimiento.

Respectodelasnuevastendencias en reorganización del contextoinstitucional, para facilitar nuevas respuestas organizativas de redes interinstitucionales nacionales e internacionales se pueden mencionar los trabajos de Mónica Casalet, Ravenna, ${ }^{7}$ quien ha desarrollado el tema en diversos trabajos, estudios sobre la innovación en las instituciones y las nuevas tendencias de información y organización que influyen en el trabajo de investigación, así como la consolidación de grupos multidisciplinarios e interinstitucionales. ${ }^{8}$ También existen trabajos como "Itinerarios del conocimiento: Formas, Dinámicas y Contenido. Un enfoque de redes". En el que se analizan las redes de generación del conocimiento, y los sectores productivos. ${ }^{9}$

\section{Perspectiva metodológica}

Por todo ello, en este trabajo se busca interpretar las nuevas políticas públicas en Ciencia y tecnología, y sus efectos en las trayectorias académicas en relación con los procesos de cambio institucional y más específicamente en el desarrollo y prácticas cotidianas de los diferentes actores involucrados. Por ello se consideró pertinente a partir de la perspectiva neo-institucionalista analizar la interacción entre los constreñimientos formales y las mediaciones valorativas configuradas en el desarrollo de los imaginarios de los distintos actores involucrados.

7 Mónica Casalet Ravenna, "Cambios en la concepción en la estructura normativa del sector de ciencia y tecnología", en Documentos de Trabajo. Serie Avances de Investigación Aportes Metodológicos 2, editado por FLACSO, 2003, México: 45.

8 Mónica Casalet Ravenna, "Cambios Institucionales para la innovación: nuevos instrumentos de política científica y tecnológica. El caso del Consorcio Xignux-CONACYT”, en Sistemas de Innovación en México. Regiones, redes y sectores editado por Daniel Villavicencio y Pedro López de Alba, 187-216. (Guanajuato, México: Plaza y Valdés, CONCYTEG y CONACYT, 2009).

9 Luna Matilde y Rosalva Casas (coord.), Itinerarios del conocimiento, formas, dinámicas y contenido. Un enfoque de redes, (México: ANTROPOS-UNAM, 2003), 34. 
En ese sentido, es un trabajo de carácter cualitativo e interdisciplinario, en la que están presentes el análisis socio-histórico, cultural e institucional. De esta manera, partimos de las diferentes aportaciones desarrolladas por autores como Burton Clark, ${ }^{10}$ los del nuevo "Institucionalismo", Powell y Di Maggio. ${ }^{11} \mathrm{Y}$ para dar cuenta de las prácticas sociales y sus representaciones simbólicas, las aportaciones de Pierre Bourdieu y Bronislaw Baczko, el sentido vivido de las prácticas, las percepciones y representaciones de los actores, respecto a su identidad y como grupo inserto en redes epistémicas.

Con esta perspectiva metodológica, nos proponemos explicar cómo los académicos y otros actores han ido construyendo un sentido de pertenencia identidad con la disciplina y la interdisciplina, con los grupos académicos y o redes epistémicas, así mismo entender las diferentes posiciones ante los cambios de las políticas actuales, a partir de sus experiencias y sus propias historias institucionales y prácticas cotidianas. En este sentido, se analizarán las políticas públicas para la construcción y consolidación de colectivos y redes a la luz de los imaginarios que se construyen por parte de los actores en relación a la participación o no en dichas redes, así como el impacto efectivo de todo ello en la actividad y la producción científica en campos del conocimiento diferentes.

A partir de una perspectiva de análisis del nuevo institucionalismo, se puede explicar la congruencia o no de las políticas institucionales en lo que respecta al fortalecimiento de la profesionalización y construcción de redes con las nuevas políticas de regulación del trabajo académico planteadas por los organismos nacionales como CONACYT, PROMEP, ANUIES e internacionales, Unesco IESALC. Sin embargo, el rescate de los imaginarios y representaciones de los distintos actores no implica desconocer la importancia de los constreñimientos y regulaciones formales para el análisis de este tema.

Por ello, en el contexto de este trabajo se definieron los constreñimientos en dos aspectos el primero, como el marco de las políticas públicas orientadas a transformar las IES y las prácticas profesionales y científicas hacia estándares de calidad y eficiencia institucional y de desempeño de los académicos, en el marco de las políticas de incentivos; y el segundo, como los distintos

\footnotetext{
10 Burton Clark, Creando Universidades Innovadoras. Estrategias organizacionales para la transformación, (México: UNAMPORRUA, 2000), 23.

11 Powell Walter y Paul Dimaggio. El nuevo institucionalismo en el análisis organizacional, (México: CNCPAP-UAEM-FCE, 1991), 45.
} 
sistemas de reglas y normas que dan especificidad a cada institución y el comportamiento de los actores.

Respecto a los primeros, las políticas actualmente aplicadas operan con una lógica de relación causa-efecto en la aplicación de incentivos para la gestión y la eficiencia y el desempeño académico, asumiendo que esto tiene validez universal para todas las IES y grupos disciplinarios.

Desde ese punto de vista, la perspectiva del Nuevo Institucionalismo nos permite observar que el cumplimiento de esta causalidad está mediado por el modo en que la oferta de los incentivos, promoción y gestión de la profesionalización académica y configuración y consolidación de las redes sea percibida y llevada a la práctica, por parte de los actores con sus diferentes imaginarios dentro de las diversas condiciones específicas en las que operan las distintas instituciones consideradas.

La dinámica de transformación de las IES y de los grupos de investigación, buscada con las políticas mencionadas, podría desembocar en resultados distintos y contradictorios con dichas políticas, sea por las diversidad de las historias de las IES, por la diversidad de las disciplinas o por el modo en que los mapas cognitivos desarrollados por los actores inciden en la definición de sus actitudes y preferencias frente a esas políticas.

Para hacer observables estas categorías, se recurre a distintos instrumentos y metodologías. En el caso de los dos constreñimientos institucionales señalados, se analizan, en primer lugar, las políticas públicas instrumentadas por parte de PROMEP y CONACYT respecto a las redes académicas. Los constreñimientos informales seanalizan con base en los diferentes imaginarios construidos por los diferentes actores, a partir de sus testimonios.

De esta manera, el estudio realizado por mí y otros investigadores que han desarrollado esta perspectiva analítica permite explicar la aparente paradoja que representa el hecho de que un número significativo de actores tenga una opinión crítica sobre los límites de las nuevas políticas, tanto en lo que se refiere a la conformación, profesionalización académica y gestión de redes de conocimiento, así como en la evaluación académica y del sistema de incentivos, y sin embargo participen en él. Esta paradoja en realidad no es tal, sino que si lo analizamos en el marco de una perspectiva 
neo-institucionalista, podría señalarse cómo las culturas formales y no formales están en una relación de tensión en los procesos de cambio institucional y social.

En el caso específico de este trabajo, el análisis de las políticas para la conformación de redes en diferentes instituciones muestra en primer lugar cómo la historia institucional, las culturas disciplinarias y la forma de organización de la planta académica determina las políticas de gestión de los grupos y redes académicas.

\section{Cambios en la legislación sobre las redes de conocimiento en México por parte de CONACYT}

En México se manifiesta también esta necesidad de reconfigurar el quehacer de la universidad en el cambio y el avance social, económico y político, por lo que en este apartado me enfocaré en hablar de la evolución de las políticas de ciencia y tecnología y, más específicamente, en aquellas políticas que incentivan la conformación de redes de conocimiento. Con la creación del CONACYT en 1970 comienza la puesta en marcha de una legislación para regular el desarrollo de esas nuevas formas de producción de conocimiento.

Algunos de los programas que establecerán con mayor énfasis el nuevo papel de las redes de conocimiento y la importancia de fomentarlas son el Programa Especial de Ciencia y Tecnología (PECyT), 2001-2006 ${ }^{12}$ y su siguiente edición 2008-2012,13 los cuales están constituidos principalmente por aportaciones del Plan Nacional de Desarrollo y el artículo 13 de la Ley para el Fomento de la Investigación Científica y Tecnológica (LFICyT). En dichos programas se menciona la importancia de apoyar la realización de proyectos de investigación aplicada con el objetivo de resolver problemas nacionales, tales como analfabetismo, pobreza, democracia, indigenismo, justicia, entre otros. Para los fines de esta investigación mencionaremos aquellos puntos que resaltan este nuevo papel de las redes de conocimiento:

- Incrementar el presupuesto nacional de actividades científicas y tecnológicas, dando prioridad a aquellos proyectos orientados a la

12 Casalet, op cit., $21-32$.

13 Casalet, op cit., 21-38. 
solución de problemas de población, respaldados por las dependencias del gobierno federal encargados de resolverlos, que involucren a grupos de investigadores, más que investigadores individuales, y que generen redes de investigación entre los diversos centros.

- Apoyar cada vez más proyectos que busquen aumentar la competitividad del sector productivo y que generen consorcios de investigación entre empresas, centros de investigación e instituciones de educación superior, sin descuidar la educación básica.

- Promover la investigación científica y tecnológica extendiendo las fronteras del conocimiento. El fortalecimiento de la economía de conocimiento plantea un cambio en la forma de producir los conocimientos y en la organización académica, ya que cada vez más se abren nuevos espacios. A los vínculos interinstitucionales se agregan las redes regionales, binacionales y multilaterales.

- Incentivar el desarrollo de nuevos campos de conocimiento.

- Promover el desarrollo y fortalecimiento de la investigación aplicada y tecnológica, ya que los cambios tecnológicos introducen nuevas configuraciones de las actividades económicas dando lugar a un aumento de sistemas locales de innovación y cadenas de valor. En cada una de estas redes de muy desigual estructura, pero vinculadas directamente al sistema productivo, se llevan a cabo procesos de creación y distribución de conocimientos que alcanzan distinto tipo de jerarquías más allá de las formas específicas que adoptan

- Fortalecer la cooperación científica y tecnológica, lo que permitirá intensificar los flujos y conocimiento entre México y otros países, una vía privilegiada son las redes internacionales de colaboración científica y tecnológica. Las transformaciones en la forma de producir, circular y apropiarse de los conocimientos apoyan un nuevo modelo no lineal de innovación, cuya clave está en las interacciones entre los agentes de muy distintos tipos (empresas, centros de investigación, universidades nacionales y extranjeras, y consultoras). ${ }^{14}$

14 CONACYT Programa Especial de Ciencia y Tecnología (PECyT), 2001-2006. 12 agosto 2013 http://dct.cicese.mx/cuaderno.pdf. $(25 / 11 / 2011)$ 
En lo que se refiere, particularmente, a la vinculación de la producción científico-tecnológica con el sector social, el Programa Especial de Ciencia y Tecnología (PECyT), 2008-2012 ${ }^{15}$ menciona varios puntos al respecto:

- Establecer políticas de Estado a corto, mediano y largo plazo que permitan fortalecer la cadena educación, ciencia básica y aplicada, tecnología e innovación, buscando generar condiciones para un desarrollo constante y una mejora en las condiciones de vida de los mexicanos.

- El Plan Nacional de Desarrollo 2007-2012 establece un conjunto de prioridades que fueron incorporadas en los programas sectorial de mediano plazo: salud, educación, alimentación, medio ambiente, agua y cambio climático, energía, crecimiento y desarrollo, combate a la pobreza, seguridad, gobernabilidad, población, equidad y género, infraestructura y turismo. En este sentido, la agenda prioritaria en el sector ciencia y tecnología establece factores fundamentales del desarrollo, en esta materia la educación de calidad y el fortalecimiento de ciencia básica y aplicada, el desarrollo tecnológico y la innovación para contribuir a mejorar el nivel de vida de la sociedad y lograr una mayor competitividad.

\section{CONCLUSIÓN}

Resultado de las entrevistas realizadas a diversos investigadores de la UNAM en el presente trabajo de investigación, el tema de las políticas actuales instrumentadas por CONACYT para la configuración de redes de conocimiento lleva a considerar varios aspectos:

Por un lado, el desarrollo no homogéneo de las estrategias para el desarrollo de esta nueva forma de organización del conocimiento en las universidades mexicanas. Cabe destacar que este ha tenido un carácter diferente y desigual. En algunas IES las nuevas estrategias de CONACYT han desempeñado un papel primordial al interior de la institución, y se detectan el desarrollo de casos exitosos ya que ha habidoun marco normativo y un plan estratégico para

15 CONACYT Programa Especial de Ciencia y Tecnología (PECyT), 2008-2012. 19 agosto 2013 http://132.247.1.49/pronaledh/pdfs/ Peciti\%202008-2012.pdf (26/11/2011). 
el flujo e intercambio de profesores quienes han participado en programas de intercambio, proyectos bilaterales de cooperación e investigaciones conjuntas por parte de diferentes grupos de investigación, es el caso de la Universidad Nacional Autónoma de México.

Pero en otros casos, se observa también que estas actividades se han llevado muchas veces a cabo por una iniciativa e interés personal de los investigadores y no necesariamente en el marco de una estrategia institucional $y$, por otro lado, es de una manera informal de pertenecer a un grupo o red de investigación.

Al no existir una política institucional $u$ oficinas especializadas en la promoción de convenios que reconozcan la existencia de redes de investigación, por la falta de definiciones conceptuales acerca de estas ni criterios que normen la acreditación de actividades académicas que se realizan por grupos de investigación o redes, se dificulta el desarrollo y consolidación de dichas redes.

Esto constituye un problema neurálgico para el desarrollo y consolidación de las redes de conocimiento, ya que al no existir una cultura institucional, en algunos casos se convierten en el desarrollo de actividades individuales y ocasionales que dificultan la permanencia de estas.

Por todo ello, se constata que los procesos de institucionalización de las redes de conocimiento, muchas veces presentan desfases entre las estrategias políticas públicas en ciencia y tecnología, y las regulaciones al interior de las IES. Por otra parte, aquellos procesos pueden no aplicarse mecánicamente en las comunidades académicas ya que están mediados por las culturas institucionales y disciplinarias diferentes, lo que dificulta más aún los procesos de configuración y desarrollo de las redes.

\section{FUENTES}

Chehaibar, Lourdes Margarita. "Entrevista personal". México Distrito Federal: 04 de abril del 2013

Andrade, Victor Manuel. "Entrevista personal". México Distrito Federal: 08 de abril del 2013 
De Teresa, Ana Paula. "Entrevista personal". México Distrito Federal: 10 de abril del 2013

Hirsch, Jorge. “Entrevista personal”. México Distrito Federal: 15 de abril del 2013

Del Rio, Fernando. “Entrevista personal”. México Distrito Federal: 22 de abril del 2013

Pozas, Ricardo. “Entrevista personal”. México Distrito Federal: 25 de abril del 2013

Hernández, Raúl. “Entrevista personal”. México Distrito Federal: 03 de mayo del 2013

Rojas, Carmen. “Entrevista personal”. México Distrito Federal: 06 de mayo del 2013

Meda, Manuel. “Entrevista personal”. México Distrito Federal: 09 de mayo del 2013

Piña, Juan Manuel. “Entrevista personal”. México Distrito Federal: 13 de mayo del 2013

Hirsch, Anita. “Entrevista personal”. México Distrito Federal: 16 de mayo del 2013

Mireles, Olivia. “Entrevista personal”. México Distrito Federal: 20 de mayo del 2013

Inclán, Catalina. “Entrevista personal”. México Distrito Federal: 12 de mayo del 2013

Montes de Oca. “Entrevista personal”. Verónica, México Distrito Federal: 11 de junio del 2013

Labastida, Julio. “Entrevista personal”. México Distrito Federal: 13 de junio del 2013

Suárez, Enrique. “Entrevista personal”. México Distrito Federal: 17 de junio del 2013

Alcantara, Armando. “Entrevista personal”. México Distrito Federal: 04 de julio del 2013

Camargo, José Luis. “Entrevista personal”. México Distrito Federal: 09 de julio del 2013

Rodríguez, Roberto. “Entrevista personal”. México Distrito Federal: 11 de julio del 2013

Ordorica, Emanuel. “Entrevista personal”. México Distrito Federal: 23 de julio del 2013

Loyo, Aurora. "Entrevista personal”. México Distrito Federal: 26 de julio del 2013Luna, Matilde. "Entrevista personal”. México Distrito Federal: 5 de agosto del 2013

Puga, Cristina. “Entrevista personal”. México Distrito Federal: 12 de agosto del 2013

Aguirre, Carlos. “Entrevista personal”. México Distrito Federal: 19 de agosto del 2013

Casas, Rosalba. “Entrevista personal”. México Distrito Federal: 26 de agosto del 2013 


\section{REFERENCIAS}

Acosta Silva, Adrián. Estado, políticas y universidades en un período de transición. Guadalajara, Jal.: FCE-UDG, 2000.

Albach, G. Philip. Educación Superior Comparada: el conocimiento de la universidad y el desarrollo. Argentina: Edit. UNESCO/UP., 2009.

Alcántara, Armando. Entre Prometeo y Sísifo. Ciencia, Tecnología y Universidad en México y Argentina. Barcelona: Pomares, 2005.

Álvarez Mendiola, Germán. Modelos académicos de Ciencias Sociales y legitimación científica en México. México: ANUIES, 2004.

ANUIES. Acciones de transformación de las universidades públicas mexicanas 1994-1999. México: ANUIES, 1999.

ANUIES. Documento estratégico para La innovación en la educación superior. México: ANUIESUPN, 2004.

Baczko, Bronislaw. Los imaginarios sociales: memorias y esperanzas colectivas. Buenos Aires: Nueva Visión, 1984.

Barnes, Barry. "Como hacer sociología del conocimiento". Política y sociedad 14/15, (19931994): 9-20.

Barquín Álvarez, Manuel et al., Tres décadas de políticas del Estado en la educación superior. México: Colección Biblioteca de la Educación Superior-ANUIES, 1998.

Bloor, David. Conocimiento e imaginario social. Barcelona: Gedisa, 2003.

Bourdieu, Pierre. Intelectuales política y poder. Argentina: Eudeba, 2000.

Brunner, José Joaquín. Los intelectuales y las Instituciones de la cultura. México: UAMANUIES, 1989.

Burton Clark. R. Creando Universidades Innovadoras. Estrategias organizacionales para la transformación. México: UNAM, Coordinación de Humanidades y Porrúa, 2000.

Casalet Ravenna, Mónica. “Cambios en la concepción en la estructura normativa del sector de ciencia y tecnología". Documentos de Trabajo. Serie Avances de Investigación Aportes Metodológicos Vol. 2. México: FLACSO, 2003.

Casalet Ravenna, Mónica. “Cambios Institucionales para la innovación: Nuevos instrumentos de política científica y tecnológica. El caso del Consorcio Xignux-CONACYT". En Sistemas de Innovación en México. Regiones, redes y sectores, editado por Daniel Villavicencio y Pedro López de Alba. Guanajuato, México: Plaza y Valdés, CONCYTEG y CONACYT, 2009. 
Casanova, Cardiel Hugo y Humberto Muñoz García. “Educación superior en México. Diferenciación y cambio hacia el fin de siglo". En Diversidad y Convergencia: Estrategias de financiamiento, gestión y reforma de la educación superior. México: CESU, 2000.

Casillas, Miguel Ángel y Adrián de Garay. “El contexto de la constitución del cuerpo académico en la Educación Superior 1960 -1990". En Colección Ensayos 37: Académicos, un botón de muestra, editado por Miguel Ángel Casillas y Adrián de Garay. México: UAM -A, 1992.

CEPAL / UNESCO. Educación y conocimiento: eje de la transformación productiva con equidad. Santiago de Chile: CEPAL-UNESCO, 1992.

Clark R, Burton. Creando Universidades Innovadoras. Estrategias organizacionales para la transformación. México: UNAM, Coordinación de Humanidades y Editorial Porrúa, 2000.

Coombs, P. H. (coord.). Estrategia para mejorar la calidad de la educación superior en México. México: SEP-FCE, 1992.

De Sierra, María Teresa y María Cristina Vera. “Nuevas identidades académicas en el horizonte histórico contemporáneo. Estudio de caso, la UNAM y la UAM". Educação, Porto Alegre Vol. 35, 2 (2012): 241-252.

De Sierra, María Teresa, (coord.). Cambio estructural y modernización educativa, México: UPNUAM- COMECSO, 1991.

De Sierra, María Teresa. “Clarososcuros de la Profesionalización Académica. Estudio de caso la UNAM y la UAM". México: UPN y Doctorado en Educación de la Ciudad de México, 2007.

De Sierra, María Teresa. "Redefinición de las relaciones Estado- IES-mercado". En Las ciencias sociales, universidad y sociedad. Temas para una agenda de posgrado, eds. Judith Bokser M. Liwerant. México: UNAM / Programa de Posgrado en Ciencias Políticas, 2003.

De Sierra, Teresa. «Politiques éducatives pour le changement des IES. Tendances internationales et nationales pendant les années 90". En L'education au regard de la mondialisation-globalisation, editado por Patricia Ducoing (coord.Gral.). Mexico: Association Francophone Internationale de Recherche Scientifique en Éducation, UNAM, CESU, 2003.

Didou Aupetit, Sylvie. Internacionalización y proveedores externos de educación superior en América Latina. México: ANUIES, 2005.

Ducoing Watty, Patricia (coord). Sujetos, actores y procesos de formación. México: SEP/CESU/ Consejo Mexicano de Investigación Educativa, 2003.

García Guadilla, Carmen. “Configuración de un nuevo perfil de prioridades para la Universidad Latinoamericana". En Cuadernos del CENDES, 20. Venezuela: Centro de Estudios del Desarrollo de la Universidad Central de Venezuela.

García Salord, Susana, Rocío Grediaga Kuri y Monique Landesmann Segall. “Los académicos en México. Hacía la constitución de un campo de conocimiento 1993-2002". En Sujetos, actores y procesos de formación editado por Patricia Ducocing Watty, (coord). México: SEP/ 
CESU / Consejo Mexicano de Investigación Educativa, 2003.

Gil Antón, Manuel (coord.). La carrera académica en la Universidad Autónoma Metropolitana: un largo y sinuoso camino. México: Grupo de Estudios sobre la Universidad, 2003.

Giménez, Gilberto, Ricardo Pozas (Coord.). Modernización e identidades sociales, México: UNAM, 1994.

Grediaga Kuri, Rocío. Profesión académica, disciplinas y organizaciones. Procesos de socialización académica y sus efectos en las actividades y resultados de los académicos mexicanos. México: Colección Biblioteca de la Educación Superior-ANUIES, 2000.

Ibarra Colado. "La nueva universidad en México: transformaciones recientes y perspectivas". Revista mexicana de investigación educativa 7, 14 (2002): 2-35.

Kent, Rollin (comp.). Los temas críticos de la educación superior en América Latina. Estudio comparativo. México: FLACSO / UAM / FCE, 1996.

Landesmann, Monique (coord.). Instituciones Educativas. Instituyendo disciplinas e identidades. México: Casa Juan Pablos, 2006.

Loyo, Aurora (coord.). Los actores sociales y la educación. Los sentidos del cambio (1988-1994). México: UNAM/Plaza y Valdés, 1997.

Luna, Matilde (coord.). Itinerarios del conocimiento: formas dinámicas y contenido. Enfoque para el análisis de redes y flujos de conocimiento. México: Editorial Anthropos, 2003.

March, James G. y Johan P. Olsen. El redescubrimiento de las instituciones. La base organizativa de la política. México: FCE y Colegio Nacional de Ciencias Políticas y Sociales y Administración Pública, 1997.

Muñoz García, Humberto (coord.). Universidad: política y cambio institucional, México: CESU-Porrúa, 2002.

Muñoz, García Humberto (coord.), Relaciones universidad-gobierno, México: UNAM-Miguel Ángel Porrúa, 2006.

Neave, Guy y Frans A. Van Vught. Prometeo Encadenado. Estado y educación superior en Europa. México: Gedisa, 1994.

Neave, Guy. Educación Superior: historia y política. Estudios comparativos sobre la universidad contemporánea. México: Editorial Gedisa, 2001.

Nord D. Estructura y cambio en la historia económica. México: Alianza Universidad, 1984.

Ordorika, Imanol (coord.). La academia en jaque. Perspectivas políticas sobre la evaluación de la educación superior en México. México: Miguel Ángel Porrúa, 2004.

Powell, Walter y Paul J. Dimaggio. The New Institutionalism in Organizational Analysis. México: CNCPAP- UAEM - FCE, 1991. 
Remedi Allione, Eduardo (coord.). Instituciones Educativas. Sujetos, historia e identidades. México: Plaza y Valdés, 2004.

Rodríguez, Gómez Roberto y Hugo Casanova Cardiel (coord.). Universidad contemporánea. Política y gobierno, Tomo II. México: CESU / UNAM / Porrúa, 1999.

Rojas Bravo, Gustavo. Modelos Universitarios. Los rumbos alternativos de la universidad y la innovación. México: UAM / FCE, 2005.

Rojas Soriano, Raúl y Amparo Ruiz del Castillo. Apuntes de la vida cotidiana: Una interpretación sociológica. México: Plaza y Valdés, 1991.

Schriewer, Jürgen. "Educación comparada: un gran programa ante nuevos desafíos". En Formación del discurso en la educación comparada compilado por Jürgen Schriewer. Barcelona: Ediciones POMARES, 2002.

Vacarezza, Silvio Leonardo. “Heterogeneidad en la conformación de la Profesión Académica: Una Comparación entre Químicos y Sociólogos". Redes 13, 26, (2007): 17-49.

Vessuri, Hebe. "Pensamiento Universitario". En La Academia va al mercado. Año 5 No 6 (1997).

GVillaseñor, Guillermo (Coord.). La identidad en la Educación Superior en México, Colección Educación Superior contemporánea. México: CESU / UNAM, 1997.

\begin{tabular}{|l|} 
\\
$\begin{array}{l}\text { De Sierra Neves, María Teresa. "Políticas públicas para la institucionalización } \\
\text { de las redes de conocimiento en las Instituciones de Educación Superior en }\end{array}$ \\
$\begin{array}{l}\text { México, desde fines de los noventa". Revista Historia de la Educación Latinoa- } \\
\text { mericana. Vol. } 16 \text { No, 22, (2014): } 231-248 .\end{array}$ \\
\hline
\end{tabular}

